\title{
Velocity vector estimation in synthetic aperture flow and B-mode imaging
}

\author{
Jensen, Jørgen Arendt
}

Published in:

IEEE International Symposium on Biomedical Imaging 2004

Link to article, DOI:

10.1109/ISBI.2004.1398467

Publication date:

2004

Document Version

Publisher's PDF, also known as Version of record

Link back to DTU Orbit

Citation (APA):

Jensen, J. A. (2004). Velocity vector estimation in synthetic aperture flow and B-mode imaging. In IEEE International Symposium on Biomedical Imaging 2004 (pp. 33-36). IEEE.

https://doi.org/10.1109/ISBI.2004.1398467

\section{General rights}

Copyright and moral rights for the publications made accessible in the public portal are retained by the authors and/or other copyright owners and it is a condition of accessing publications that users recognise and abide by the legal requirements associated with these rights.

- Users may download and print one copy of any publication from the public portal for the purpose of private study or research.

- You may not further distribute the material or use it for any profit-making activity or commercial gain

- You may freely distribute the URL identifying the publication in the public portal

If you believe that this document breaches copyright please contact us providing details, and we will remove access to the work immediately and investigate your claim. 


\title{
VELOCITY VECTOR ESTIMATION IN SYNTHETIC APERTURE FLOW AND B-MODE IMAGING
}

\author{
Jørgen Arendt Jensen \\ Center for Fast Ultrasound Imaging, Ørsted•DTU, Bldg. 348, \\ Technical University of Denmark, DK-2800 Kgs. Lyngby, Denmark
}

\begin{abstract}
A method for determining both velocity magnitude and angle in a synthetic aperture ultrasound system is described. The approach uses directional beamforming along the flow direction and cross-correlation to determine velocity magnitude. The angle of the flow is determined from the maximum normalized correlation calculated as a function of angle. The method is investigated using data measured by an experimental ultrasound scanner from a flow rig. A commercial $7 \mathrm{MHz}$ linear array transducer is used and data are measured for flow angles of $60^{\circ}$ and $90^{\circ}$. The velocity magnitude is determined with a precision of $0.36 \%\left(60^{\circ}\right)$ and $1.2 \%\left(90^{\circ}\right)$, respectively. The $60^{\circ}$ angle is estimated with a bias of $0.54^{\circ}$ and a standard deviation of $2.1^{\circ}$. For $90^{\circ}$ the bias is $0.0003^{\circ}$ and standard deviation $1.32^{\circ}$.
\end{abstract}

\section{INTRODUCTION}

Current ultrasound velocity estimation systems only estimate the blood velocity projected onto the direction of the steered ultrasound beam [1]. The velocities are, thus, only found in one direction in the image, and velocities in the direction perpendicular to the beam cannot be estimated. Neither the correct velocity magnitude nor the velocity angle are, thus, estimated.

A correct velocity estimation system should be able to give both velocity magnitude and direction. A directional beamforming approach was suggested for conventional ultrasound imaging in [2] and for synthetic aperture (SA) flow imaging in [3]. Here the received ultrasound beam is focused along the flow direction and the correct velocity magnitude is thereby determined. The angle for the beam processing is, however, manually determined from the B-mode image before beamforming.

This paper will present a method for also estimating the velocity angle in a SA flow system. The procedure determines both magnitude and angle using cross-correlation of the received signals, and it can be employed for estimation

This work was supported by grant 9700883,9700563 and 26-01-0178 from the Danish Science Foundation and by B-K Medical A/S, Denmark. of both blood velocity and tissue motion. The basic principle of directional synthetic aperture flow imaging is described in Section 2 and the angle determination procedure is detailed in Section 3. The performance of the approach is determined in Section 4 from data measured using an experimental ultrasound scanner and a flow rig.

\section{METHOD FOR SYNTHETIC APERTURE FLOW IMAGING}

SA ultrasound images are acquired by emitting a spherical wave with one or a collection of transducer elements [4, 5]. The scattered signals are then received by all transducer elements, and a low resolution image is generated by focusing the received signal in all points of the image. The process is repeated for other sets of transmitting elements and new low resolution images are formed. Combining all low resolution images will give a high resolution image, that is dynamically focused in both transmit and receive, thus yielding a higher resolution than traditional images, if a sufficient number of transmissions $N_{t}$ are used [6]. A new high resolution image can be formed after each transmission, if the transmission sequence is repeated. The oldest transmission event is then replaced by the newest, and the imaging can therefore be done recursively [7].

SA images can also be used for flow estimation, if the data collection is repeated. Data from two high resolution images can be correlated to obtain the velocity of the blood or tissue [8]. It is here important that the two images are acquired in a short time interval, and that the emission sequence is exactly the same to maximize correlation. The recursive image formation can also be employed here and a new correlation function estimate formed after each emission. It is here important that the image formation sequence is exactly the same and high resolution images $N_{t}$ emissions apart should only be correlated. The correlation functions can, however, be averaged for all emissions, if the velocity is constant [8].

SA flow imaging has several advantages compared to traditional ultrasound flow imaging. The prime advantage is the continuously available data for all points in the image. 


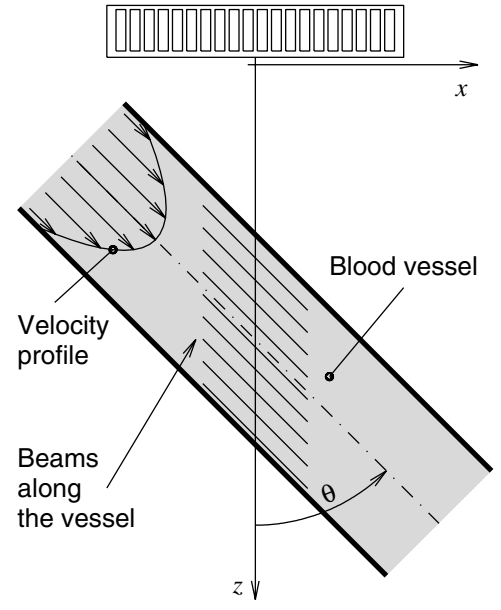

Fig. 1. Beamforming is made along the laminar flow.

The velocity estimation can therefore take place continuously and the initialization of echo canceling filters and the limitation on the number of samples for velocity estimation are eliminated.

Another advantage of SA flow imaging is that data can be focused in any direction. It is, thus, possible to focus signals along the flow direction as suggested in [3] and shown in Fig. 1 The high resolution image data are then beamformed along the direction of the flow. The focusing points are given by $\vec{r}_{p}(k)=\left[\Delta r \cdot k \cdot \sin (\theta), 0, \Delta r \cdot k \cdot \cos (\theta)+z_{s t}\right]$, where $\Delta r$ is spatial sampling interval, $k$ sample index, $\theta$ flow angle between the flow vector and $z$-axis, and $z_{s t}$ depth of the vessel. The data are focused for each emission and the final directionally signal is obtained by adding all low resolution images $L_{j}$ :

$$
y_{d}(k)=\sum_{j=1}^{N_{t}} L_{j}\left(\vec{r}_{p}(k)\right) .
$$

The spatial movement between high resolution images is $\overrightarrow{d_{s}}=\vec{v} T_{p r f} N_{t}$, where the blood velocity is $\vec{v}=|\vec{v}|[\sin \theta, 0$, $\cos \theta]$. This distance corresponds to a sample index of:

$$
k_{s}=\frac{|\vec{v}| T_{p r f} N_{t}}{\Delta r}
$$

Correlating two received signals over $N_{k}+1$ samples from two high resolution images gives

$$
\begin{aligned}
R_{12}(l) & =\frac{1}{N_{k}+1} \sum_{k=-N_{k} / 2}^{N_{k} / 2} y_{d}^{(n)}(k) y_{d}^{\left(n+N_{t}\right)}(k+l) \\
& =\frac{1}{N_{k}+1} \sum_{k=-N_{k} / 2}^{N_{k} / 2} y_{d}^{(n)}(k) y_{d}^{(n)}\left(k+l-k_{s}\right) \\
& =R_{11}\left(l-k_{s}\right)
\end{aligned}
$$

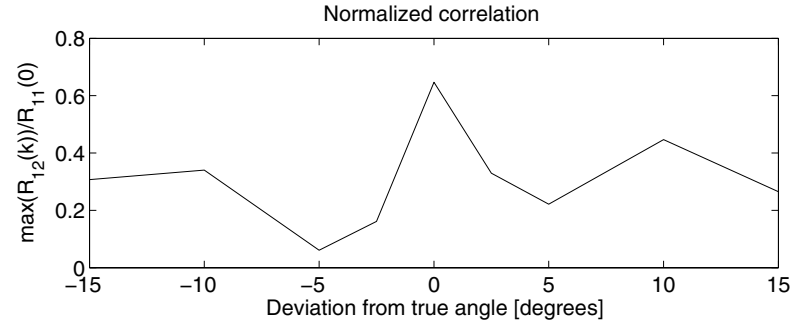

Fig. 2. Normalized maximum correlation as a function of beam formation angle.

where $y_{d}^{(n)}(k)$ is the directional signal focused after emission $n$ and $R_{11}(l)$ is its autocorrelation function.

A global maximum is found at $l=k_{s}$ and the velocity magnitude is estimated from:

$$
|\vec{v}|=\frac{k_{s} \Delta r}{T_{p r f} N_{t}} .
$$

Often $\Delta r$ is large to keep the amount of beam formation low, and parabolic interpolation [9] is used to increase the precision of the estimated velocity.

\section{DETERMINATION OF VELOCITY ANGLE}

The velocity angle for beam formation has previously been determined manually from the B-mode image, and this Section introduces an automatic approach for determining the angle.

The primary reason for decorrelation of traditional flow signals is the velocity distribution within the range gate. This makes part of the scatterers travel faster or slower than others, which decorrelates the received signal from emission to emission. The directional beamforming avoids the decorrelation, since it tracks the scatterers in the correct direction and thereby maintains a high correlation. This observation is used to devise a method for automatic angle determination. The correlation peak found for the correct angle normalized by the power of the signal must therefore have the highest correlation values, since it has the least decorrelation due to a velocity distribution. This is stated as:

$$
\begin{aligned}
R_{12 n}\left(\theta_{m}\right) & =\left(\frac{\max \left(R_{12}\left(l, \theta_{m}\right)\right)}{R_{11}\left(0, \theta_{m}\right)}\right) \\
\theta & =\underset{\theta_{m}}{\operatorname{Arg} \min } R_{12 n}\left(\theta_{m}\right)
\end{aligned}
$$

where $\max \left(R_{12}\left(l, \theta_{m}\right)\right)$ is the maximum value of the crosscorrelation function for a given angle and $R_{11}\left(0, \theta_{m}\right)$ is the corresponding power of the signal. The correct angle $\theta$ is found where the normalized correlation function as a function of angle $R_{12 n}\left(\theta_{m}\right)$ has its peak value. 


\begin{tabular}{|l|c|c|}
\hline Center frequency & $f_{0}$ & $7 \mathrm{MHz}$ \\
\hline Wavelength & $\lambda=c / f_{0}$ & $0.22 \mathrm{~mm}$ \\
\hline Element pitch & $w$ & $0.208 \mathrm{~mm}$ \\
\hline Element height & $h_{e}$ & $4.5 \mathrm{~mm}$ \\
\hline Number of receive elements & $N_{r}$ & 128 \\
\hline Number of transmit events & $N_{t}$ & 8 \\
\hline Elevation focus & $R_{e}$ & $20 \mathrm{~mm}$ \\
\hline \hline Directional signals & $N_{e}$ & 128 \\
\hline Pulse repetition frequency & $f_{p r f}$ & $3 \mathrm{kHz}$ \\
\hline RF sampling frequency & $f_{s}$ & $40 \mathrm{MHz}$ \\
\hline Distance between estimates & $d z$ & $0.25 \mathrm{~mm}$ \\
\hline Sampling interval for lines & $\Delta r=\lambda / 20$ & $0.011 \mathrm{~mm}$ \\
\hline Correlation interval & $-10 \lambda: 10 \lambda$ & $-2.2: 2.2 \mathrm{~mm}$ \\
\hline \hline Radius of vessel & $R$ & $8.5 \mathrm{~mm}$ \\
\hline Distance to vessel center & $z_{s t}$ & $37.5 \mathrm{~mm}$ \\
\hline Peak velocity in flow & $v_{0}$ & $0.15 \mathrm{~m} / \mathrm{s}$ \\
\hline
\end{tabular}

Table 1. Standard parameters for transducer and parabolic flow measurement.

An example of the calculated normalized correlation is shown in Fig. 2 for a true angle of $90^{\circ}$. The data are from the measurements described in the next Section. The correlation has been calculated for every $5^{\circ}$. The deviation from the true angle is shown, and the global maximum coincides with the correct angle.

The resolution in angle determination is here equal to $5^{\circ}$, which is less accurate than desired. An interpolated value using a parabolic approximation of the peak is calculated for the angle as

$$
\begin{aligned}
\theta_{\text {int }} & =\theta- \\
& \frac{R_{12 n}(\theta+1)-R_{12 n}(\theta-1)}{2\left(R_{12 n}(\theta+1)-2 R_{12 n}(\theta)+R_{12 n}(\theta-1)\right)} \Delta \theta
\end{aligned}
$$

to avoid having to calculate the directional signals for too many directions. Here $\Delta \theta$ is the angle sampling interval. The number of values can of course be narrowed down, if prior information can be used to determine a rough estimate. This could be from either temporal or spatial neighbors to the current estimate.

\section{RESULTS}

The angle determination method was investigated using a circulating flow rig. A Smedegaard EcoWatt 1 pump circulates a blood mimicking fluid made by Danish Phantom Service consisting of water, glycerol, orgasol, Trition x100 , NaBenzoat, and $\mathrm{K}_{2}$ EDTA diluted 10 to 1 with demineralised water. A reduction valve was used to control the velocity. The tubing consisted of a $1.2 \mathrm{~m}$ long, $20 \mathrm{~mm}$ diameter steel tube before the flow entered a $18 \mathrm{~mm}$ diameter heat shrink tubing. The thickness of the heat shrink tubing

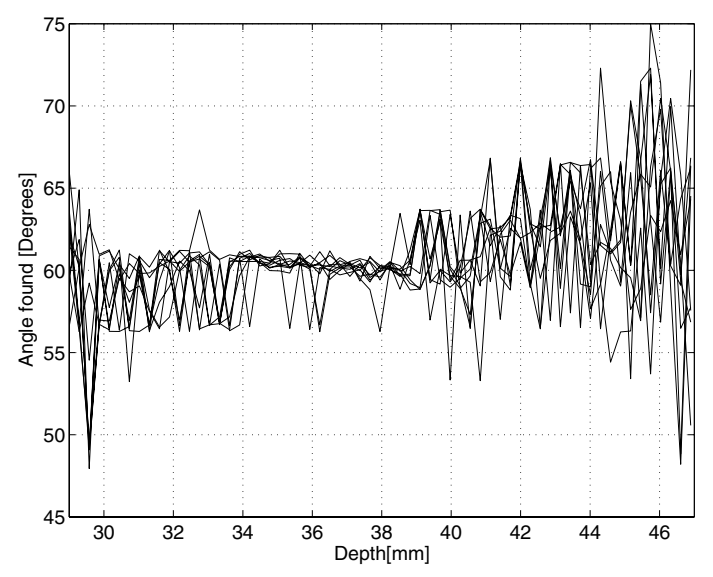

Fig. 3. Estimated velocity angles for a true velocity angle of $60^{\circ}$.

was $0.5 \mathrm{~mm}$ giving an internal diameter of $17 \mathrm{~mm}$. The flow was maintained with a peak velocity less than $0.15 \mathrm{~m} / \mathrm{s}$ giving a Reynolds number of 1,276 assuming a viscosity of 2 $\mathrm{cP}$ and a parabolic profile.

Measurements were performed using the RASMUS research scanner [10]. It can emit arbitrary signals in 128 individual channels and can simultaneously measure 64 receive channels at $40 \mathrm{MHz}$ with 12 bits precision. A 2-to-1 multiplexing makes it possible to cover all 128 transducer elements in 2 emissions. Data are stored in the system's 16 GBytes of memory in real-time, and they are transferred to a Linux computer cluster with $32 \mathrm{CPUs}$ for beam formation and velocity estimation.

A $7 \mathrm{MHz}$ commercial linear array transducer was used in the experiments. The measurements have been performed by using 8 emissions equally spread over the 128 element aperture, with each emission using 11 defocused elements to emulate a spherical wave emission [4]. A frequency encoded chirp is used in transmission to increase the signalto-noise ratio $[11,6]$. The chirp has a duration of $20 \mu \mathrm{s}$ and a bandwidth of $7 \mathrm{MHz}$ centered around the transducer center frequency. The receiving elements are multiplexed to be closest to the emitting center element, and all 128 receiving elements are hereby sampled. The transmission is repeated with a pulse repetion frequency of $3 \mathrm{kHz}$ and 3,000 individual emissions have been acquired by repeating the sequence 375 times. A summary of the parameters used for data acquisition and processing is shown in Table 1.

The received RF element signals are first matched filtered to compress the signals in the axial direction and increase the signal-to-noise ratio [11]. The resulting signals are focused in direction of the flow forming low resolution directional signals. The high resolution signals are made by adding the latest 8 low resolution signals. Stationary echo canceling is performed by finding the mean value of the focused lines and then subtracting this from the signals. 


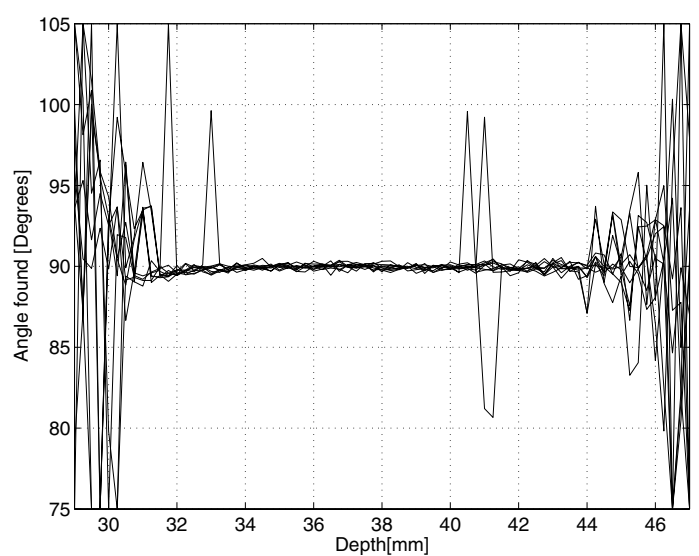

Fig. 4. Estimated velocity angles for a true velocity angle of $90^{\circ}$.

The focused directional signals from the same image formation are subsequently cross-correlated and added to the other cross-correlation functions, and the velocity is found from the combined cross-correlation function.

Two experiments at flow angles of 60 degrees and 90 degrees (transverse velocity) were performed. The velocity was found from 16 sequences of 8 emissions, each corresponding to a total of 128 emissions. This is the same number of emissions that is used in normal spectral velocity imaging [1] over which the flow normally can be considered quasi-stationary in the human body.

The velocity could be estimated with a standard deviation over the whole profile of $0.36 \%$ relative to the peak velocity of the vessel for a velocity angle of $60^{\circ}$. The standard deviation was $1.2 \%$ for $90^{\circ}[3]$.

The angle determination method has been applied on the data and the results are shown in Fig. 3 for a true velocity angle of $60^{\circ}$. One line is shown for each of the 20 independent velocity estimates. The vessel boundaries are between 30 and $46 \mathrm{~mm}$. Here the mean value of all estimates is $60.54^{\circ}$ and the standard deviation is $2.1^{\circ}$. A slight increase in standard deviation as a function of depth is seen. This is probably due to the decrease in signal-to-noise ratio as a function of depth.

The same experiment has been repeated for a true velocity angle of $90^{\circ}$ and the result are shown in Fig. 4. Here the mean value is $90.0003^{\circ}$ and the standard deviation is $1.32^{\circ}$.

\section{CONCLUSION}

An approach for determining both velocity magnitude and angle in a SA flow system has been presented. For data measured using an experimental ultrasound scanner and a flow rig both magnitude and angle could be determined with a relative standard deviation of roughly $1 \%$ for flow angles of $60^{\circ}$ and $90^{\circ}$.

\section{REFERENCES}

[1] J. A. Jensen, Estimation of Blood Velocities Using Ultrasound: A Signal Processing Approach, Cambridge University Press, New York, 1996.

[2] J. A. Jensen and I. R. Lacasa, "Estimation of blood velocity vectors using transverse ultrasound beam focusing and cross-correlation," in Proc. IEEE Ultrason. Symp., 1999, pp. 1493-1497.

[3] J. A. Jensen and S. I. Nikolov, "Transverse flow imaging using synthetic aperture directional beamforming," in Proc. IEEE Ultrason. Symp., 2002, pp. 1488-1492.

[4] M. Karaman, P. C. Li, and M. O'Donnell, "Synthetic aperture imaging for small scale systems," IEEE Trans. Ultrason., Ferroelec., Freq. Contr., vol. 42, pp. 429-442, 1995.

[5] G. R. Lockwood, J. R. Talman, and S. S. Brunke, "Real-time 3-D ultrasound imaging using sparse synthetic aperture beamforming," IEEE Trans. Ultrason., Ferroelec., Freq. Contr., vol. 45, pp. 980-988, 1998.

[6] K. L. Gammelmark and J. A. Jensen, "Multielement synthetic transmit aperture imaging using temporal encoding," IEEE Trans. Med. Imag., vol. 22, no. 4, pp. 552-563, 2003.

[7] S. I. Nikolov, K. Gammelmark, and J. A. Jensen, "Recursive ultrasound imaging," in Proc. IEEE Ultrason. Symp., 1999, vol. 2, pp. 1621-1625.

[8] S. I. Nikolov and J. A. Jensen, "In-vivo synthetic aperture flow imaging in medical ultrasound," IEEE Trans. Ultrason., Ferroelec., Freq. Contr., pp. 848856, 2003.

[9] S. G. Foster, A pulsed ultrasonic flowmeter employing time domain methods, Ph.D. thesis, Dept. Elec. Eng., University of Illinois, Urbana, Ill., 1985.

[10] J. A. Jensen, O. Holm, L. J. Jensen, H. Bendsen, H. M. Pedersen, K. Salomonsen, J. Hansen, and S. Nikolov, "Experimental ultrasound system for real-time synthetic imaging," in Proc. IEEE Ultrason. Symp., 1999, vol. 2, pp. 1595-1599.

[11] T. X. Misaridis and J. A. Jensen, "An effective coded excitation scheme based on a predistorted FM signal and an optimized digital filter," in Proc. IEEE Ultrason. Symp., 1999, vol. 2, pp. 1589-1593. 